\title{
On the Density of Real Quadratic Fields with $\lambda_{2}=\mu_{2}=v_{2}=0$
}

\author{
Hisao TAYA ${ }^{1, *}$ and Gen YAMAMOTO ${ }^{2, *}$ \\ ${ }^{1}$ Miyagi University of Education, Department of Mathematics, \\ Aramaki-Aza-Aoba 149, Aoba-ku, Sendai 980-0845, Japan \\ ${ }^{2}$ Tokyo Denki University, School of Science and Technology for Future Life, \\ Kanda Nishiki-cho 2-2, Chiyoda-ku, Tokyo 101-8457, Japan
}

Received February 13, 2009; final version accepted May 22, 2009

\begin{abstract}
According to the previous works by Nakagawa-Horie and the first author, the density of real quadratic fields with $\lambda_{3}=\mu_{3}=v_{3}=0$ is greater than or equal to $\frac{17}{24}$. In this note, however, we will report the density of real quadratic fields with $\lambda_{2}=\mu_{2}=v_{2}=0$ to be zero.
\end{abstract}

KEYWORDS: quadratic fields, Iwasawa invariants, density

\section{Introduction}

For a number field $k$ and a prime number $\ell$, we denote by $A(k)$ the $\ell$-Sylow subgroup of the ideal class group of $k$, by $k_{\infty}$ the cyclotomic $\mathbb{Z}_{\ell}$-extension of $k$, and by $k_{n}$ the $n$-th layer in $k_{\infty} / k$. Then Iwasawa [Iw59] proved that there exist three integers $\lambda_{\ell}=\lambda_{\ell}(k) \geq 0, \mu_{\ell}=\mu_{\ell}(k) \geq 0$ and $v_{\ell}=v_{\ell}(k)$, depending only on $k$ and $\ell$, such that

$$
\# A\left(k_{n}\right)=\ell^{\lambda_{\ell} n+\mu_{\ell} \ell^{n}+v_{\ell}}
$$

for every sufficiently large $n$. Here, \#S means the cardinality of $S$ for a finite set $S$. These integers $\lambda_{\ell}(k), \mu_{\ell}(k)$ and $\nu_{\ell}(k)$ are called the (cyclotomic) Iwasawa $\lambda$-, $\mu$ - and $\nu$-invariants, respectively, of $k$ for $\ell$.

Following Iwasawa's mention in [Iw73, page 316], Greenberg proposed in [Gr76] that it would be $\lambda_{\ell}(k)=\mu_{\ell}(k)=0$ for any totally real number field $k$ and any prime number $\ell$. This is now known as Greenberg's conjecture. As for the $\mu$-invariant, Ferrero-Washington [FW79] proved that $\mu_{\ell}(k)=0$ for any abelian (not necessarily totally real) number field $k$ and any prime number $\ell$. However, very little is known about $\lambda_{\ell}(k)$ for totally real number fields $k$ except for $k=\mathbb{Q}$, even in the case of real quadratic fields.

Ono [On99] and Byeon [By01, By03] proved that, for any prime number $\ell \geq 5$, there are infinitely many real quadratic fields $k$ with $\lambda_{\ell}(k)=\mu_{\ell}(k)=v_{\ell}(k)=0$ by giving an estimate for the number of such $k$. However at present, in the case where $\ell \geq 5$, we have no good estimate on the density of real quadratic fields $k$ with $\lambda_{\ell}(k)=\mu_{\ell}(k)=$ $v_{\ell}(k)=0$ in all real quadratic fields. On the other hand, when $\ell=3$, Nakagawa-Horie [NH88] and the first author [Ta00] showed that the density of real quadratic fields $k$ with $\lambda_{3}(k)=v_{3}(k)=v_{3}(k)=0$ is greater than or equal to $\frac{17}{24}$.

Theorem 1.1 (Nakagawa-Horie [NH88], Taya [Ta00]). For each real quadratic field $k$, let $d_{k}$ denote the discriminant of $k$. Then we have the following:

$$
\liminf _{x \rightarrow \infty} \frac{\#\left\{k: \text { real quad. } \mid d_{k}<x, \lambda_{3}(k)=\mu_{3}(k)=v_{3}(k)=0\right\}}{\#\left\{k: \text { real quad. } \mid d_{k}<x\right\}} \geq \frac{17}{24} .
$$

In this note, we will report the case $\ell=2$. The result is as follows, which says that the density of real quadratic fields $k$ with $\lambda_{2}(k)=\mu_{2}(k)=v_{2}(k)=0$ is equal to zero.

Theorem 1.2. For each real quadratic field $k$, let $d_{k}$ denote the discriminant of $k$. Then we have the following:

$$
\lim _{x \rightarrow \infty} \frac{\#\left\{k: \text { real quad. } \mid d_{k}<x, \lambda_{2}(k)=\mu_{2}(k)=v_{2}(k)=0\right\}}{\#\left\{k: \text { real quad. } \mid d_{k}<x\right\}}=0 .
$$

To show Theorem 1.2, we use the following theorem.

Theorem 1.3. Let $k$ be a real quadratic field. Then $\lambda_{2}(k)=\mu_{2}(k)=v_{2}(k)=0$ if and only if $k$ is one of the following:

*Corresponding author. E-mail: taya@staff.miyakyo-u.ac.jp; gen@cck.dendai.ac.jp 
(i) $k=\mathbb{Q}(\sqrt{p})$, where $p$ is a prime number such that

(i-1) $p=2$, or

(i-2) $p \equiv 3(\bmod 4)$, or

(i-3) $p \equiv 5(\bmod 8)$, or

(i-4) $p \equiv 1(\bmod 8)$ and $\left(\frac{2}{p}\right)_{4}\left(\frac{p}{2}\right)_{4}=-1$.

(ii) $k=\mathbb{Q}(\sqrt{2 p})$, where $p$ is a prime number satisfying (i-2), (i-3) or (i-4).

(iii) $k=\mathbb{Q}(\sqrt{p q})$, where $p$ and $q$ are distinct prime numbers such that

(iii-1) $p \equiv q \equiv 3(\bmod 8)$, or

(iii-2) $p \equiv 3, q \equiv 7(\bmod 8)$.

(iv) $k=\mathbb{Q}(\sqrt{2 p q})$, where $p$ and $q$ are distinct prime numbers satisfying (iii-1) or (iii-2).

Here, $\left(\frac{*}{*}\right)_{4}$ denotes the biquadratic residue symbol defined by $\left(\frac{2}{p}\right)_{4} \equiv 2^{\frac{p-1}{4}}(\bmod p)$ and by $\left(\frac{p}{2}\right)_{4}=1$ or -1 according as $p \equiv 1$ or $9(\bmod 16)$, for prime numbers $p$ satisfying $p \equiv 1(\bmod 8)$.

Theorem 1.3 is an immediate consequence of Theorem 2.4 in [TY06]. However, we have not given the detail of the proof of Theorem 2.4 in [TY06] and it is still unpublished anywhere. So, in $\$ 2$, we will give an elementary proof of Theorem 1.3 for the completeness of this paper; the proof is different from what we have explained briefly in [TY06]. In $\S 3$, we will show Theorem 1.2 by using Theorem 1.3. Further, we will introduce the results on the case of imaginary quadratic fields when $\ell=2$ in $\S 4$.

Finally, we mention a result on the vanishing of all Iwasawa invariants in the case of odd primes $\ell$. In [Ya00], the second author determined all (real) abelian $\ell$-extension fields over $\mathbb{Q}$ with $\lambda_{\ell}(k)=\mu_{\ell}(k)=v_{\ell}(k)=0$ for any odd prime $\ell$. Theorem 2.4 in [TY06] is a " $\ell=2$ " version of this result, and the idea of its proof is the same as in the case of odd primes, though its proof is more complicated than the case of odd primes.

\section{The proof of Theorem 1.3}

In this section, we will give an elementary proof of Theorem 1.3.

Let $m$ be a positive square-free integer and $k=\mathbb{Q}(\sqrt{m})$. We consider the case $\ell=2$, so $k_{\infty}$ is the cyclotomic $\mathbb{Z}_{2^{-}}$ extension of $k$. When $m=2$, we have $k=\mathbb{Q}(\sqrt{2})=\mathbb{Q}_{1}$. So we already knew that $\lambda_{2}(k)=\mu_{2}(k)=v_{2}(k)=0$. Hence, we get the case (i-1) of (i) in Theorem 1.3. Therefore, in the rest of this section, we assume that $m \neq 2$.

For $k=\mathbb{Q}(\sqrt{m})$, put $k^{\prime}=\mathbb{Q}(\sqrt{2 m})$. Then we have $k_{\infty}=k_{\infty}^{\prime}$, and $k_{1}=k_{1}^{\prime}=\mathbb{Q}(\sqrt{m}, \sqrt{2})$. We use the notation through this section.

First, we have the following:

Proposition 2.1. $\lambda_{2}(k)=\mu_{2}(k)=v_{2}(k)=0$ if and only if $A\left(k_{1}\right)$ is trivial.

Proof. Note that $k_{\infty} / k$ or $k_{\infty} / k^{\prime}$ is totally ramified at 2 . If $k_{\infty} / k$ is totally ramified at 2 , we get easily that $\# A\left(k_{n}\right) \leq \# A\left(k_{n+1}\right)$ for all integers $n \geq 0$. Hence $\lambda_{2}(k)=\mu_{2}(k)=v_{2}(k)=0$ if and only if $A\left(k_{n}\right)=0$ for all integers $n \geq 0$. By a theorem of Fukuda [Fu94, Theorem 1], the latter assertion is equivalent to $A\left(k_{1}\right)=0$. If $k_{\infty} / k^{\prime}$ is totally ramified at 2 , we have $A\left(k_{1}^{\prime}\right)=A\left(k_{1}\right)=0$ by the same argument.

Remark 2.2. Let $K$ be a number field and $\ell$ a prime number which totally ramifies in $K_{\infty} / K$. Then Proposition 2.1 also holds for $K$ and $\ell$.

According to the value of $m$, we divide our arguments into the following cases:

(a) $m \equiv 1(\bmod 8)$,

(b) $m \equiv 5(\bmod 8)$,

(c) $m \equiv 3(\bmod 4)$,

(d) $m \equiv 2(\bmod 4)$ and $\frac{m}{2} \equiv 1(\bmod 8)(m \neq 2)$,

(e) $m \equiv 2(\bmod 4)$ and $\frac{m}{2} \equiv 5(\bmod 8)$,

(f) $m \equiv 2(\bmod 4)$ and $\frac{m}{2} \equiv 3(\bmod 4)$.

Then the following proposition holds.

Proposition 2.3. We have the following:

(i) In the case of (b), (c) and (f), $\lambda_{2}(k)=\mu_{2}(k)=v_{2}(k)=0$ if and only if $2 \chi h(k)$.

(ii) In the case of (e), $\lambda_{2}(k)=\mu_{2}(k)=v_{2}(k)=0$ if and only if $2 \times h\left(k^{\prime}\right)$.

(iii) In the case of (a), $\lambda_{2}(k)=\mu_{2}(k)=v_{2}(k)=0$ if and only if $2 \| h\left(k^{\prime}\right)$.

(iv) In the case of (d), $\lambda_{2}(k)=\mu_{2}(k)=\nu_{2}(k)=0$ if and only if $2 \| h(k)$.

Here, $h(k)$ (resp. $\left.h\left(k^{\prime}\right)\right)$ is the class number of $k$ (resp. $\left.k^{\prime}\right)$.

Proof. (i) and (ii): In the cases (b), (c) and (f) (resp. (e)), $k_{\infty} / k$ (resp. $\left.k_{\infty} / k^{\prime}\right)$ is totally ramified at 2 . Hence, $\lambda_{2}(k)=\mu_{2}(k)=v_{2}(k)=0$ if and only if $A\left(k_{n}\right)$ (resp. $A\left(k_{n}^{\prime}\right)$ ) is trivial for any $n \geq 0$. Further, since 2 does not split in $k$ (resp. $\left.k^{\prime}\right)$, the assertion follows from a theorem of Iwasawa [Iw56]. 
(iii) and (iv): In the case of (a) (resp. (d)), $k_{1} / k^{\prime}$ (resp. $k_{1} / k$ ) is unramified. Hence, by [OT97, Lemma 1], $2 \| h\left(k^{\prime}\right)$ (resp. $2 \| h(k)$ ) if and only if $A\left(k_{1}\right)$ is trivial. So, the assertion follows from Proposition 2.1.

Now we prove Theorem 1.3 by using the above proposition.

Proof of Theorem 1.3. Let $d_{k}$ be the discriminant of $k, r(k)$ the 2-rank of the ideal class group of $k$, and $r^{*}(k)$ the 2-rank of the narrow ideal class group of $k$. It is well known that if $d_{k}$ has an odd prime divisor $p$ such that $p \equiv 3(\bmod 4)$ then $r(k)=r^{*}(k)-1$, otherwise $r(k)=r^{*}(k)$ (cf. [SW88]). And we also know that the 2-rank and 4-rank of the narrow ideal class group of $k$ can be calculated by genus theory and the theorem of Rédei and Reichardt (cf. [RR33]). These are main tools we use below.

First, in the case of (b), (c) and (f), we see by genus theory and the theorem of Rédei and Reichardt that $m$ with $2 \nmid h(k)$ are as follows:

(b1) $m=p$ is a prime such that $p \equiv 5(\bmod 8)$,

(b2) $m=p q$, where $p$ and $q$ are primes such that $p \equiv 3, q \equiv 7(\bmod 8)$,

(c) $m=q$ is a prime such that $q \equiv 3(\bmod 4)$,

(f) $m=2 q$, where $q$ is a prime such that $q \equiv 3(\bmod 4)$.

And, in the case of (e), we see by genus theory and the theorem of Rédei and Reichardt that $m$ with $2 \nmid h\left(k^{\prime}\right)$ are as follows:

(e1) $m=2 p$, where $p$ is a prime such that $p \equiv 5(\bmod 8)$,

(e2) $m=2 p q$, where $p$ and $q$ are primes such that $p \equiv 3, q \equiv 7(\bmod 8)$.

Hence, we obtain the case (i-2), (i-3) of (i) and (ii), and the case (iii-2) of (iii) and (iv) in Theorem 1.3.

Next, we consider the case of (a). Then $d_{k^{\prime}}=8 \mathrm{~m}$. When $m$ has at least two prime divisors, we see by genus theory and the theorem of Rédei and Reichardt that a composite number $m$ with $2 \| h\left(k^{\prime}\right)$ is as follows:

(a1) $m=p q$, where $p$ and $q$ are distinct primes such that $p \equiv q \equiv 3(\bmod 8)$.

When $m$ is prime, Proposition 3.4 in [Ya84] says that $\left(\frac{2}{p}\right)_{4}\left(\frac{p}{2}\right)_{4}=-1$ if and only if $2 \| h\left(k^{\prime}\right)$. So, a prime number $m$ with $2 \| h\left(k^{\prime}\right)$ is as follows:

(a2) $m=p$ is a prime such that $p \equiv 1(\bmod 8)$ and $\left(\frac{2}{p}\right)_{4}\left(\frac{p}{2}\right)_{4}=-1$.

Hence, we obtain the case (i-4) of (i) and the case (iii-1) of (iii) in Theorem 1.3.

Finally, we consider the case of (d). We denote by $m=2 m^{\prime}$, where $m^{\prime} \neq 1$. Then $d_{k}=8 m^{\prime}$. Again, we see by the same way as in the case of (a) that $m$ with $2 \| h(k)$ are as follows:

(d1) $m=2 p q$, where $p$ and $q$ are distinct primes such that $p \equiv q \equiv 3(\bmod 8)$.

(d2) $m=2 p$ is a prime such that $p \equiv 1(\bmod 8)$ and $\left(\frac{2}{p}\right)_{4}\left(\frac{p}{2}\right)_{4}=-1$.

Hence, we obtain the case (iii-1) of (iv), and (i-4) of (ii) in Theorem 1.3.

Therefore this concludes the proof of Theorem 1.3.

\section{The proof of Theorem 1.2}

In this section, we will show Theorem 1.2. First, we will estimate the number of real quadratic fields satisfying conditions in Theorem 1.3.

For any real number $x>0$, we put

$$
\mathcal{K}_{(s-t)}^{(u)}(x):=\left\{k=\mathbb{Q}(\sqrt{m}) \mid d_{k}<x, m \text { satisfying }(s-t) \text { of }(u) \text { in Theorem } 1.3\right\} .
$$

For example, when $(s-t)=(\mathrm{i}-3)$ and $(u)=($ ii $)$, we have

$$
\begin{aligned}
\mathcal{K}_{(\mathrm{i}-3)}^{(\mathrm{ii})}(x) & :=\left\{k=\mathbb{Q}(\sqrt{m}) \mid d_{k}<x, m \text { satisfying (i-3) of (ii) in Theorem } 1.3\right\} \\
& =\left\{k=\mathbb{Q}(\sqrt{2 p}) \mid d_{k}<x, p \equiv 5(\bmod 8): \text { prime }\right\} .
\end{aligned}
$$

Then the following holds, except the case corresponding to the condition (i-4):

Proposition 3.1. We have the following:

(1) $\# \mathcal{K}_{(\mathrm{i}-1)}^{(\mathrm{i})}(x)=1$ if $x \gg 0$,

(2) $\# \mathcal{K}_{(\mathrm{i}-2)}^{(\mathrm{i})}(x) \sim \frac{x}{8 \log x} \quad(x \rightarrow \infty)$,

(3) $\# \mathcal{K}_{(\mathrm{i}-3)}^{(\mathrm{i})}(x) \sim \frac{x}{4 \log x} \quad(x \rightarrow \infty)$,

(4) $\# \mathcal{K}_{(\mathrm{i}-2)}^{(\mathrm{ii})}(x) \sim \frac{x}{16 \log x} \quad(x \rightarrow \infty)$,

(5) $\# \mathcal{K}_{(\mathrm{i}-3)}^{(\mathrm{ii})}(x) \sim \frac{x}{32 \log x} \quad(x \rightarrow \infty)$,

(6) $\# \mathcal{K}_{\text {(iii-1) }}^{\text {(iii) }}(x) \sim \frac{x(\log \log x)}{16 \log x} \quad(x \rightarrow \infty)$,

(7) $\# \mathcal{K}_{\text {(iii-2) }}^{\text {(iii) }}(x) \sim \frac{x(\log \log x)}{8 \log x} \quad(x \rightarrow \infty)$,

(8) $\# \mathcal{K}_{\text {(iii-1) }}^{\text {(iv) }}(x) \sim \frac{x(\log \log x)}{128 \log x} \quad(x \rightarrow \infty)$,

(9) $\# \mathcal{K}_{\text {(iii-2) }}^{\text {(iv) }}(x) \sim \frac{x(\log \log x)}{64 \log x} \quad(x \rightarrow \infty)$.

Here, for two functions $f$ and $g$ of $x, f \sim g(x \rightarrow \infty)$ means $\lim _{x \rightarrow \infty} \frac{f}{g}=1$. 
Remark 3.2. At present, we do not get the exact estimate of the number of real quadratic fields satisfying (i-4) of (i) and (ii) in Theorem 1.3, namely, $\# \mathcal{K}_{(\mathrm{i}-4)}^{(\mathrm{i})}(x)$ and $\# \mathcal{K}_{(\mathrm{i}-4)}^{(\mathrm{ii})}(x)$. However, the following upper bounds of these are easily obtained by the same way as in the proof of Proposition 3.1:

$$
\begin{aligned}
& \# \mathcal{K}_{(\mathrm{i}-4)}^{(\mathrm{i})}(x) \leq \frac{x}{4 \log x} \quad \text { for } x \gg 0, \\
& \# \mathcal{K}_{(\mathrm{i}-4)}^{(\mathrm{ii})}(x) \leq \frac{x}{32 \log x} \quad \text { for } x \gg 0 .
\end{aligned}
$$

Proof. (1) This is trivial because $\mathcal{K}_{(\mathrm{i}-1)}^{(\mathrm{i})}(x)=\{\mathbb{Q}(\sqrt{2})\}$ if $x \gg 0$.

(2) In this case, it follows that

$$
\begin{aligned}
\# \mathcal{K}_{(\mathrm{i}-2)}^{(\mathrm{i})}(x) & =\#\left\{k=\mathbb{Q}(\sqrt{p}) \mid d_{k}<x, p \equiv 3(\bmod 4): \text { prime }\right\} \\
& =\#\left\{p: \operatorname{prime} \mid p<\frac{x}{4}, p \equiv 3(\bmod 4)\right\} \\
& \sim \frac{1}{\varphi(4)} \frac{\frac{x}{4}}{\log \frac{x}{4}} \sim \frac{x}{8 \log x} \quad(x \rightarrow \infty)
\end{aligned}
$$

from the prime number theorem and Dirichlet's theorem on primes in arithmetic progressions. Here, $\varphi$ is the Euler $\varphi$-function.

(3) (5) In these cases, the assertions follow from the same way as in (2).

(6) In this case, it follows that

$$
\begin{aligned}
\# \mathcal{K}_{(\mathrm{iii}-1)}^{(\mathrm{iii})}(x) & =\#\left\{k=\mathbb{Q}(\sqrt{p q}) \mid d_{k}<x, p \equiv q \equiv 3(\bmod 8): \text { primes }\right\} \\
& =\#\{p q: \text { product of two primes } \mid p q<x, p \equiv q \equiv 3(\bmod 8)\} \\
& \sim \frac{1}{\varphi(8)} \frac{1}{\varphi(8)} \frac{x(\log \log x)}{\log x} \sim \frac{x(\log \log x)}{16 \log x} \quad(x \rightarrow \infty)
\end{aligned}
$$

from the prime number theorem on products of two primes (cf. [HW08, Theorem 437]) and Dirichlet's theorem on primes in arithmetic progressions.

(7) (9) In these cases, the assertions follow from the same way as in (6).

Now we give the proof of Theorem 1.2.

Proof of Theorem 1.2. For any real number $x>0$, we denote by $\mathcal{K}(x)$ the set of real quadratic fields with discriminant $d_{k}<x$. Then, as is well known (cf. [NH88]), we have

$$
\# \mathcal{K}(x) \sim \frac{3 x}{\pi^{2}} \quad(x \rightarrow \infty),
$$

where $\pi$ is the circular constant.

On the other hand, Proposition 3.1 and Remark 3.2 say that, for each (s-t) of (u) except (i-1) of (i), we have one of the following:

$$
\# \mathcal{K}_{(s-t)}^{(u)}(x) \sim c_{1} \frac{x}{\log x} \quad(x \rightarrow \infty)
$$

or

$$
\# \mathcal{K}_{(s-t)}^{(u)}(x) \sim c_{2} \frac{x(\log \log x)}{\log x} \quad(x \rightarrow \infty),
$$

where $c_{1}$ and $c_{2}$ are constants. Theorem 1.3 implies that

$$
\left\{k \in \mathcal{K}(x) \mid \lambda_{2}(k)=\mu_{2}(k)=v_{2}(k)=0\right\}=\bigcup_{(s-t) \text { of }(u)} \mathcal{K}_{(s-t)}^{(u)}(x) .
$$

Therefore, it follows

$$
\lim _{x \rightarrow \infty} \frac{\#\left\{k \in \mathcal{K}(x) \mid \lambda_{2}(k)=\mu_{2}(k)=v_{2}(k)=0\right\}}{\# \mathcal{K}(x)} \leq \lim _{x \rightarrow \infty} \frac{c \frac{x}{\log x}+c^{\prime} \frac{x(\log \log x)}{\log x}+1}{\frac{3 x}{\pi^{2}}}=0,
$$

where $c$ and $c^{\prime}$ are constants. Hence we get the conclusion.

\section{The case of imaginary quadratic fields}

Finally, we mention the case of imaginary quadratic fields when $\ell=2$. Though the contents in this section are more or less introduced in [Ho96], we will reconsider the density of imaginary quadratic fields with $\lambda_{2}(k)=\mu_{2}(k)=0$ by paying our attention on $v$-invariants. 
For a real number $x>0$, we denote by $\mathcal{K}^{-}(x)$ the set of imaginary quadratic fields whose discriminant $>-x$. Then the following proposition follows from Kida's formula (cf. [Ki79]) and a theorem of Iwasawa [Iw56]:

Proposition 4.1. Let $k$ be an imaginary quadratic field. Then $\lambda_{2}(k)=\mu_{2}(k)=v_{2}(k)=0$ if and only if $k$ is one of the following:

(i) $k=\mathbb{Q}(\sqrt{-1})$,

(ii) $k=\mathbb{Q}(\sqrt{-2})$,

(iii) $k=\mathbb{Q}(\sqrt{-p})$,

(iv) $k=\mathbb{Q}(\sqrt{-2 p})$,

where $p$ is a prime number satisfying $p \equiv 3(\bmod 8)$.

Hence, since as is well known

$$
\# \mathcal{K}^{-}(x) \sim \frac{3 x}{\pi^{2}} \quad(x \rightarrow \infty)
$$

we get the following by Dirichlet's theorem on primes in arithmetic progressions and the prime number theorem:

Proposition 4.2. We have

$$
\lim _{x \rightarrow \infty} \frac{\#\left\{k \in \mathcal{K}^{-}(x) \mid \lambda_{2}(k)=\mu_{2}(k)=v_{2}(k)=0\right\}}{\# \mathcal{K}^{-}(x)}=0 .
$$

In the case of imaginary quadratic fields, we also determine all imaginary quadratic fields with $\lambda_{2}(k)=\mu_{2}(k)=0$ and $v_{2}(k)>0$ by Kida's formula (cf. [Ki79]) and a theorem of Iwasawa [Iw56]. The result is as follows:

Proposition 4.3. Let $k$ be an imaginary quadratic field. Then $\lambda_{2}(k)=\mu_{2}(k)=0$ and $v_{2}(k)>0$ if and only if $k$ is one of the following:

(i) $k=\mathbb{Q}(\sqrt{-p})$,

(ii) $k=\mathbb{Q}(\sqrt{-2 p})$,

where $p$ is a prime number satisfying $p \equiv 5(\bmod 8)$. And further, in both cases (i) and (ii), we obtain $k_{\infty}=\mathbb{Q}_{\infty}(\sqrt{-p})$, $\lambda_{2}(k)=\mu_{2}(k)=0$ and $v_{2}(k)=1$.

By the same reason of Proposition 4.2, we have

$$
\lim _{x \rightarrow \infty} \frac{\#\left\{k \in \mathcal{K}^{-}(x) \mid \lambda_{2}(k)=\mu_{2}(k)=0, \nu_{2}(k)>0\right\}}{\# \mathcal{K}^{-}(x)}=0 .
$$

Hence, we have the following:

Proposition 4.4 (Horie [Ho96]). We have

$$
\lim _{x \rightarrow \infty} \frac{\#\left\{k \in \mathcal{K}^{-}(x) \mid \lambda_{2}(k)=\mu_{2}(k)=0\right\}}{\# \mathcal{K}^{-}(x)}=0 .
$$

From this, as for Greenberg's conjecture, we see the assumption that $k$ is totally real to be essential (though we may carry owls to Athens).

\section{REFERENCES}

[By01] Byeon, D., "Indivisibility of class numbers and Iwasawa $\lambda$-invariants of real quadratic fields," Compositio Math., 126: 249-256 (2001).

[By03] Byeon, D., "Existence of certain fundamental discriminants and class numbers of real quadratic fields," J. Number Theory, 98: 432-437 (2003).

[FW79] Ferrero, B., and Washington, L. C., "The Iwasawa invariant $\mu_{p}$ vanishes for abelian number fields," Ann. of Math., 109: 377-395 (1979).

[Fu94] Fukuda, T., "Remarks on $\mathbb{Z}_{p}$-extensions of Number fields," Proc. Japan Acad., 70: 264-266 (1994).

[Gr76] Greenberg, R., "On the Iwasawa invariants of totally real number fields,” Amer. J. Math., 98: 263-284 (1976).

[HW08] Hardy, G. H., and Wright, E. M., An Introduction to the Theory of Numbers, 6th Ed., Oxford University Press (2008).

[Ho96] Horie, K., "Iwasawa invariant" (Japanese), Sūgaku, 48: 358-371 (1996).

[Iw56] Iwasawa, K., "A note on class numbers of algebraic number fields," Abh. Math. Sem. Hamburg, 20: 257-258 (1956).

[Iw59] Iwasawa, K., “On Г-extensions of algebraic number fields," Bull. Amer. Math. Soc., 65: 183-226 (1959).

[Iw73] Iwasawa, K., "On $\mathbb{Z}_{l}$-extensions of algebraic number fields," Ann. of Math., 98: 246-326 (1973).

[Ki79] Kida, Y., "On cyclotomic $\mathbb{Z}_{2}$-extensions of imaginary quadratic field," Tôhoku Math. J. (2), 31: 91-96 (1979).

[NH88] Nakagawa, J., and Horie, K., "Elliptic curves with no rational points," Proc. Amer. Math. Soc., 104: 20-24 (1988).

[On99] Ono, K., "Indivisibility of class numbers of real quadratic fields," Compositio Math., 119: 1-11 (1999).

[OT97] Ozaki, M., and Taya, H., "On the Iwasawa $\lambda_{2}$-invariants of certain families of real quadratic fields," Manuscripta Math. Soc., 94: 437-444 (1997). 
[RR33] Rédei, L., and Reichardt, H., "Die Anzahl der durch 4 teilbaren Invarianten der Klassengruppe eines beliebigen quadratischen Zahlkörpers," J. Reine Angew. Math., 170: 69-74 (1933).

[Ta00] Taya, H., "Iwasawa invariants and class numbers of quadratic fields for the prime 3," Proc. Amer. Math. Soc., 128: 12851292 (2000).

[SW88] Saito, M., and Wada, H., "Tables of ideal class groups of real quadratic fields," Proc. Japan Acad., 64A: $347-349$ (1988).

[TY06] Taya, H., and Yamamoto, G., Notes on certain real abelian 2-extension fields with $\lambda_{2}=v_{2}=v_{2}=0$, Trends in Math., Information Center for Mathematical Sciences, KAIST, 9: 81-89 (2006).

[Ya00] Yamamoto, G., "On the vanishing of Iwasawa invariants of absolutely abelian p-extensions," Acta. Arith., 94: 365-371 (2000).

[Ya84] Yamamoto, Y., "Divisibility by 16 of class number of quadratic fields whose 2-class groups are cyclic," Osaka J. Math., 21: 1-22 (1984). 